\title{
Knowledge, attitudes and potential response to menu labelling in an urban public health clinic population
}

\author{
Jennifer Piron ${ }^{1}$, Lisa $\bigvee$ Smith ${ }^{1,3}$, Paul Simon ${ }^{2,3}$, Patricia L Cummings ${ }^{4}$ and Tony Kuo ${ }^{4,5, *}$ \\ ${ }^{1}$ Surveillance, Epidemiology and Evaluation (SEE), Division of Chronic Disease and Injury Prevention, Los \\ Angeles County Department of Public Health, Los Angeles, CA, USA: ${ }^{2}$ Division of Chronic Disease and Injury \\ Prevention, Los Angeles County Department of Public Health, Los Angeles, CA, USA: ${ }^{3}$ UCLA School of Public \\ Health, Los Angeles, CA, USA: ${ }^{4}$ Office of Senior Health, Division of Chronic Disease and Injury Prevention, \\ Los Angeles County Department of Public Health, 3530 Wilshire Blvd, Los Angeles, CA 90010, USA: \\ ${ }^{5}$ Department of Family Medicine, UCLA David Geffen School of Medicine, Los Angeles, CA, USA
}

Submitted 24 March 2009: Accepted 17 July 2009: First published online 26 August 2009

\begin{abstract}
Objective: The present study examines the receptivity to and potential effects of menu labelling on food choices of low-income and minority individuals - a group often at disproportionate risk for preventable, lifestyle-related health conditions (e.g. obesity, diabetes and CVD).

Design: We conducted a cross-sectional survey to examine the knowledge, attitudes and potential response to menu labelling in an urban public health clinic population.

Setting: United States.

Subjects: A total of 639 clinic patients were recruited in the waiting rooms of six, large public health centres in Los Angeles County (2007-2008). These centres provide services to a largely uninsured or under-insured, low-income, Latino and African-American population.

Results: Among those approached and who met eligibility criteria, $88 \%$ completed the survey. Of the 639 respondents, $55 \%$ were overweight or obese based on selfreported heights and weights; $74 \%$ reported visiting a fast food restaurant at least once in the past year, including $22 \%$ at least once a week; $93 \%$ thought that calorie information was 'important'; and $86 \%$ thought that restaurants should be required to post calorie information on their menu boards. In multivariate analyses, respondents who were obese, female, Latino and supportive of calorie postings were more likely than others to report that they would choose food and beverages with lower calories as a result of menu labelling.

Conclusions: These findings suggest that clinic patients are receptive to this population-based strategy and that they would be inclined to change their food selections in response to menu labelling.
\end{abstract}

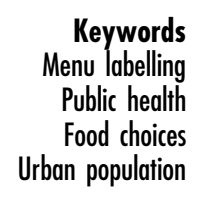

The passage of Senate Bill (SB) $1420^{(1)}$ in California marks a milestone in public policy by requiring all restaurant chains across the state with twenty or more facilities to post calorie information next to each item on their menus and menu boards. SB 1420 builds upon previous menu labelling efforts in other local jurisdictions, including New York City, San Francisco and Seattle-King County, Washington. These regulatory efforts have been developed to combat the rising obesity epidemic in the USA ${ }^{(2-5)}$.

In 2008, researchers at the Los Angeles County Department of Public Health conducted a health impact assessment of menu labelling to assess the potential impact of this legislative strategy on population weight gain in this populous county ${ }^{(3)}$. They found that if $10 \%$ of restaurant patrons ordered reduced calorie meals as a result of calorie postings at the point of purchase, with an average reduction of 100 calories per meal, then menu labelling as specified in SB 1420 would avert at least $37 \cdot 3 \%$ of the 6.75 million pound average annual weight gain in the County population aged 5 years and older ${ }^{(3)}$. The study included a sensitivity analysis that indicated a potentially greater impact if calorie postings in restaurants were more widely accepted and utilised by the public. This would be particularly true if groups most severely affected by the obesity epidemic, including low-income, Latino and African-American populations, were responsive to these menu postings. However, to the best of our knowledge, little is known about the knowledge, attitudes and the potential response of these populations to calorie postings on menus and menu boards. 
To address this gap, we surveyed a sample of patients at public health clinics in Los Angeles County, CA, USA. The population served by these clinics is predominantly low-income, uninsured or under-insured, and disproportionately Latino and African-American.

\section{Methods}

\section{Survey and study area}

The Calorie and Nutrition Information Survey (CNIS), a cross-sectional survey of public health clinic patients in Los Angeles County, was conducted between 2007 and 2008. Participants were recruited from six public health centres operated by the Los Angeles County Department of Public Health and located within underserved areas of the County (across six zip codes). Compared with the County overall, these areas, on average, have more overcrowded housing $(32 \% v .23 \%)^{(6)}$; increased prevalence of child obesity $(27 \cdot 8 \% v \cdot 23 \cdot 3 \%)^{(7)}$; and higher poverty rate $(25 \cdot 7 \% \text { v. } 17 \cdot 9 \%)^{(6)}$. Fast food restaurants were also known to be located in close proximity to public schools in these areas ${ }^{(8)}$.

\section{Recruitment and questionnaire}

The participants were recruited in the waiting rooms of each of the clinics during pre-specified data collection periods, the recruitment procedures accounted for seasonal as well as daily variation in patient volume. A systematic, serial sampling protocol (i.e. first 100 patients visiting each clinic on a pre-determined date) was employed to screen for eligibility and for participation in the CNIS. Eligibility criteria included, at each health centre: (i) patients of the tuberculosis, sexually transmitted disease (STD) and/or immunisation clinics; (ii) young adults 15-17 years of age attending the STD clinic or adults, 18 years and older, attending any of the three clinics; and (iii) individuals who speak English or Spanish. The 15-17 year age group was included because this group represents an important subgroup of STD clinics (albeit not a large proportion of the total clinic visits); the age limit for receiving care without parental consent at these clinics is 12 years and older. Inclusion and exclusion of the 15-17 year age group during subsequent analyses yielded no statistically significant differences in the final results.

Among those who approached and met eligibility criteria, $88 \%$ of them ( $n 639$, total) completed the study instrument in English or Spanish. The self-administered questionnaire consisted of questions about respondent characteristics including age, gender, race/ethnicity, height and weight, health-related lifestyle behaviours, knowledge of daily caloric requirements, frequency of restaurant visitations, food selection intentions and attitudes toward calorie postings at the point of purchase. No information about the state menu labelling law ${ }^{(1)}$ or visual aids such as photos of food items were shown to survey participants. Most question items in the questionnaire were developed using previously validated questions from Centers for Disease Control and Prevention (CDC)-sponsored population surveys including the National Health Interview Survey and the National Health and Nutrition Examination Survey. Other question items were adapted from existing research instruments found in the literature ${ }^{(9)}$.

All participants gave informed consent prior to completing the thirteen-question, multiple-item questionnaire. Prior to fieldwork, all study protocols were reviewed and approved by the Los Angeles County Department of Public Health Institutional Review Board.

\section{Defining overweight/obesity}

BMI was calculated using self-reported height and weight. For adults aged 18 years and above, overweight and obesity were defined as BMI between 25.0 and 29.9 and $\geq 30 \cdot 0 \mathrm{~kg} / \mathrm{m}^{2}$, respectively; for younger adults aged $15-17$ years, CDC growth charts were used to determine overweight (BMI-for-age near 95th percentile) and obesity (BMI-for-age $\geq 95$ th percentile) $^{(2)}$.

\section{Data analyses}

Descriptive analyses were performed to generate frequency distributions of the survey variables. Univariate and bivariate analyses were performed to generate crude odds ratios and $95 \%$ confidence limits used in subsequent analyses of the relationships between the independent and dependent variables (i.e. receptivity to menu labelling and food selection intentions). Nationally recommended age- and gender-specific caloric requirements for sedentary and moderately active young adults and adults aged 18 years and above ${ }^{(10)}$ were used to determine whether a respondent reported correct knowledge about his/her daily caloric requirements. One of the variables, the question concerning the degree of importance of having calorie information posted on menu boards (i.e. degree of receptivity to menu labelling), was dichotomised into two categories: 'important' (i.e. very important, important and somewhat important) $v$. 'not important at all'. The primary outcome variable in the multivariate analysis - intention to order foods with 'less calories' if point-of-purchase calorie postings were available (potential response to menu labelling) - was also dichotomised into two categories: would order foods and drinks with 'less calories' $v$. with 'same calories'.

The Health Belief Model ${ }^{(11)}$ was adapted to help guide the variable selection and the multivariate analysis of factors predicting food selection intentions if calorie information was posted at the point of purchase. Logistic regression analyses were employed to calculate adjusted odds ratios (AOR) for hypothesised predictors of these self-reported intentions. Independent variables were entered simultaneously into the model, and assessment of model fit was computed using the Hosmer-Lemeshow Goodness-of-Fit test. All statistical analyses were performed using SAS version 9·1 (SAS Institute, Cary, NC, USA). 


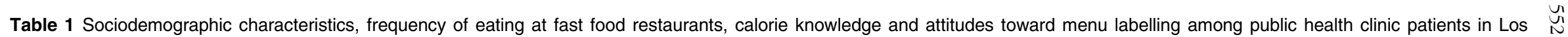
Angeles County $(n$ 639)

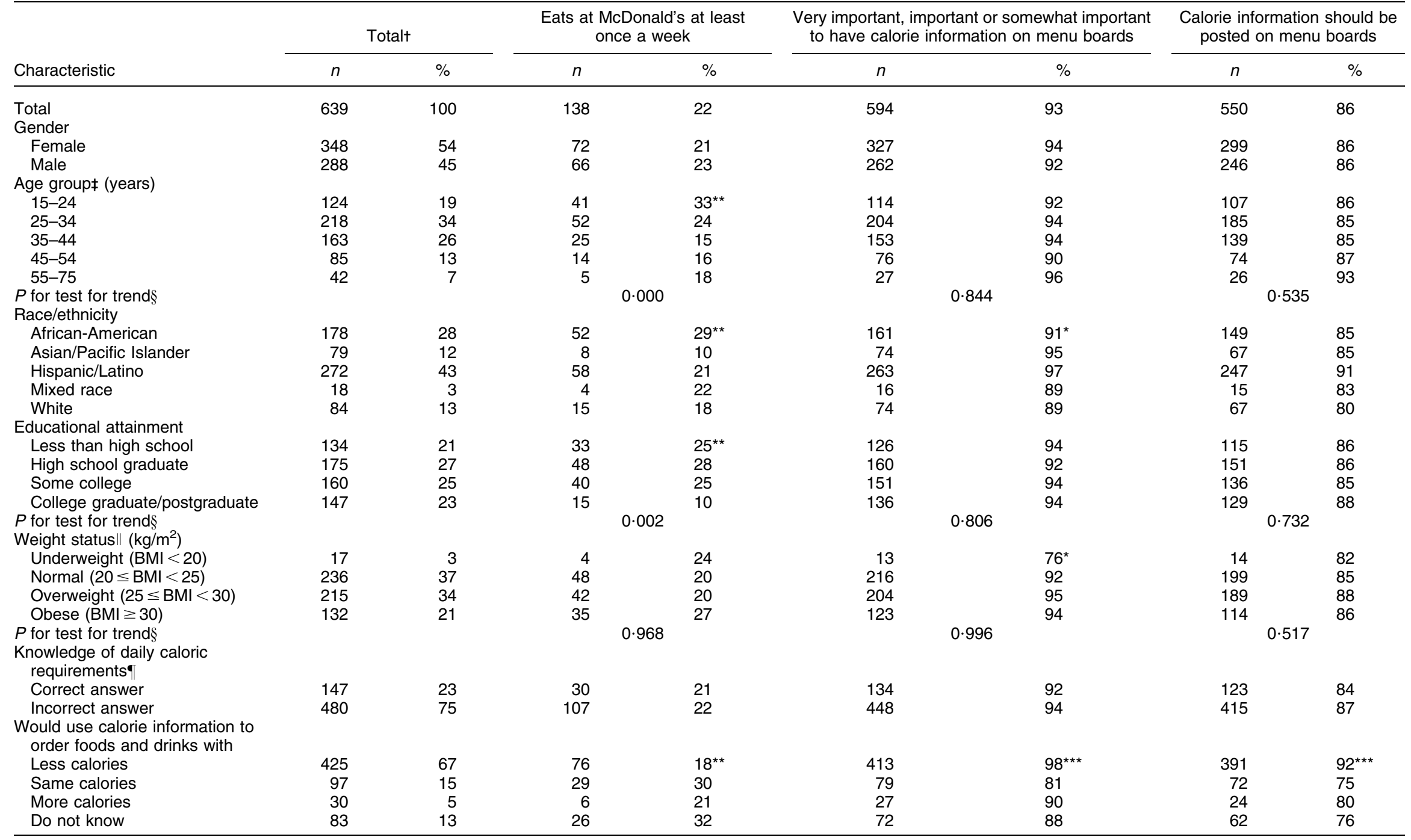

Calorie and Nutrition Information Survey, 2007-2008

${ }^{\star} P<0.05 ;{ }^{* \star} P<0.01 ;{ }^{\star \star \star} P<0.001 ; P$ values derived by Pearson's $\chi^{2}$ test; Fisher's exact test was used for cell counts less than five. tValues may not sum up to $100 \%$ due to missing or rounded numbers.

$\neq$ Mean age $=34.9($ sD 11.6$)$ years.

$P$ values derived from Cochran-Armitage test for linear trend (two-tailed).

As defined by the Centers for Disease Control and Prevention BMl estimates and growth charts. BMI calculations in the study are based on self-reported heights and weights.

-The number of calories the respondent should consume based on his/her age, height and weight as indicated in the US Department of Agriculture Nutrition Pyramid (April 2005). 


\section{Results}

\section{Eating out, knowledge of caloric requirements and menu labelling receptivity}

The mean age of the respondent sample was 34.9 (SD 11.6) years. The sample was predominantly female, Latino and African-American, and included a relatively high percentage of individuals (55\%) who were overweight and obese (see Table 1). In the past year, $74 \%$ of them reported eating out, having visited a well-known fast food restaurant (McDonald's), with 22\% going there at least once a week; the frequency of visits was the highest among individuals less than 45 years of age and among those with less than a high school education. Only 19\% who visited McDonald's in the past year remembered seeing some form of calorie information posted. Seventy-five per cent were unable to correctly report the number of calories that they should be consuming in a single day per US Department of Agriculture (USDA) guidelines ${ }^{(10)}$. This finding is confirmed by other studies that suggest that most Americans, even among trained nutritionists, typically underestimate their daily caloric intake or are unaware of the requirements ${ }^{(5,12)}$.

Table 2 Predictors of using calorie information on restaurant menu boards to order foods items with less calories ( $v$. same calories) among public health clinic patients in Los Angeles County

\begin{tabular}{|c|c|c|c|c|c|c|}
\hline \multirow[b]{2}{*}{ Characteristic } & \multicolumn{2}{|c|}{ Totalt } & \multicolumn{2}{|c|}{ Crude odds ratio (OR) } & \multicolumn{2}{|c|}{ Adjusted odds ratio (AOR) $\ddagger$} \\
\hline & $n$ & $\%$ & OR & $95 \% \mathrm{CL}$ & AOR & $95 \% \mathrm{CL}$ \\
\hline $\begin{array}{l}\text { Total } \\
\text { Gender }\end{array}$ & 639 & 100 & - & - & - & - \\
\hline Female & 348 & 54 & $1 \cdot 6$ & $1 \cdot 0,2 \cdot 6^{*}$ & $1 \cdot 8$ & $1 \cdot 1,3 \cdot 0^{*}$ \\
\hline Male & $\begin{array}{l}340 \\
288\end{array}$ & $\begin{array}{l}34 \\
45\end{array}$ & 1.0 & - & $1 \cdot 0$ & - \\
\hline \multicolumn{7}{|l|}{ Age (years) } \\
\hline $15-24$ & 124 & 19 & $1 \cdot 0$ & - & $1 \cdot 0$ & - \\
\hline $25-34$ & 218 & 34 & $2 \cdot 3$ & $1 \cdot 2,4 \cdot 2^{*}$ & $2 \cdot 4$ & $1 \cdot 2,4 \cdot 8^{*}$ \\
\hline $35-44$ & 163 & 26 & $1 \cdot 6$ & $0.9,3 \cdot 0$ & $1 \cdot 4$ & $0 \cdot 7,2 \cdot 9$ \\
\hline $45-54$ & 85 & 13 & $1 \cdot 4$ & $0 \cdot 6,2 \cdot 9$ & 1.5 & $0 \cdot 6,3 \cdot 5$ \\
\hline $55-75$ & 42 & 7 & $4 \cdot 6$ & $1 \cdot 0,21 \cdot 0^{*}$ & $7 \cdot 0$ & $1 \cdot 3,39 \cdot 4^{*}$ \\
\hline \multicolumn{7}{|l|}{ Race/ethnicity } \\
\hline African-American & 178 & 28 & $1 \cdot 4$ & $0 \cdot 7,2 \cdot 6$ & $1 \cdot 6$ & $0 \cdot 7,3 \cdot 4$ \\
\hline Asian/Pacific Islander & 79 & 12 & $2 \cdot 1$ & $1 \cdot 0,4 \cdot 7^{\star}$ & $1 \cdot 7$ & $0 \cdot 7,4 \cdot 4$ \\
\hline Hispanic/Latino & 272 & 43 & $3 \cdot 7$ & $1 \cdot 9,7 \cdot 1^{*}$ & $3 \cdot 4$ & $1 \cdot 5,8 \cdot 0^{\star}$ \\
\hline Mixed race & 18 & 3 & $3 \cdot 2$ & $0 \cdot 7,15 \cdot 3$ & $2 \cdot 3$ & $0 \cdot 4,11 \cdot 8$ \\
\hline White & 84 & 13 & $1 \cdot 0$ & - & $1 \cdot 0$ & - \\
\hline \multicolumn{7}{|l|}{ Education } \\
\hline Less than high school & 134 & 21 & 1.5 & $0 \cdot 7,3 \cdot 2$ & $1 \cdot 2$ & $0.4,3 \cdot 4$ \\
\hline High school graduate & 175 & 27 & $0 \cdot 7$ & $0 \cdot 4,1 \cdot 4$ & 0.5 & $0 \cdot 2,1 \cdot 1$ \\
\hline Some college & 160 & 25 & 0.7 & $0 \cdot 4,1 \cdot 3$ & $0 \cdot 6$ & $0 \cdot 3,1 \cdot 3$ \\
\hline College graduate/postgraduate & 147 & 23 & $1 \cdot 0$ & - & $1 \cdot 0$ & - \\
\hline \multicolumn{7}{|l|}{ Weight status $\S\left(\mathrm{kg} / \mathrm{m}^{2}\right)$} \\
\hline Underweight (BMI < 20) & 17 & 3 & $1 \cdot 5$ & $0 \cdot 3,7 \cdot 2$ & $1 \cdot 0$ & $0 \cdot 2,5 \cdot 9$ \\
\hline Overweight $(25 \leq \mathrm{BMI}<30)$ & 215 & 34 & $1 \cdot 8$ & $1 \cdot 1,2 \cdot 9^{\star}$ & $1 \cdot 4$ & $0 \cdot 8,2 \cdot 6$ \\
\hline Obese $(\mathrm{BMI} \geq 30)$ & 132 & 21 & $2 \cdot 6$ & $1 \cdot 3,5 \cdot 1^{*}$ & $2 \cdot 3$ & $1 \cdot 1,5 \cdot 0^{\star}$ \\
\hline Normal $(20 \leq \mathrm{BMI}<25)$ & 236 & 37 & 1.0 & - & $1 \cdot 0$ & - \\
\hline \multicolumn{7}{|c|}{ Knowledge of daily caloric requirements\| } \\
\hline Correct answer & 147 & 23 & $1 \cdot 0$ & - & $1 \cdot 0$ & - \\
\hline Incorrect answer & 480 & 75 & 0.8 & $0 \cdot 5,1 \cdot 4$ & $0 \cdot 8$ & $0 \cdot 4,1 \cdot 5$ \\
\hline \multicolumn{7}{|l|}{ Ate at McDonald's } \\
\hline At least once a week & 138 & 22 & 0.5 & $0 \cdot 2,1 \cdot 0$ & 0.5 & $0 \cdot 2,1 \cdot 1$ \\
\hline At least once a month & 177 & 28 & $1 \cdot 0$ & $0 \cdot 5,2 \cdot 0$ & $0 \cdot 7$ & $0 \cdot 3,1 \cdot 6$ \\
\hline Few times a year & 158 & 25 & $1 \cdot 0$ & $0 \cdot 5,2 \cdot 0$ & $0 \cdot 9$ & $0.4,1.9$ \\
\hline Almost never/never & 163 & 26 & $1 \cdot 0$ & - & $1 \cdot 0$ & - \\
\hline \multicolumn{7}{|l|}{ Calorie information on menu boards } \\
\hline Somewhat important or better & 594 & 93 & $8 \cdot 9$ & $3 \cdot 8,20 \cdot 8^{\star}$ & $6 \cdot 6$ & $2 \cdot 4,18 \cdot 2^{*}$ \\
\hline Not at all important & 42 & 7 & $1 \cdot 0$ & - & $1 \cdot 0$ & - \\
\hline \multicolumn{7}{|c|}{$\begin{array}{l}\text { Calorie information should be posted on menu } \\
\text { boards next to food items }\end{array}$} \\
\hline Yes & 550 & 86 & $3 \cdot 5$ & $1 \cdot 9,6 \cdot 2^{*}$ & $2 \cdot 4$ & $1 \cdot 1,4 \cdot 9^{\star}$ \\
\hline No & 87 & 14 & $1 \cdot 0$ & - & $1 \cdot 0$ & - \\
\hline
\end{tabular}

Calorie and Nutrition Information Survey, 2007-2008.

$\mathrm{CL}$, confidence limits.

${ }^{\star} P<0.05$.

tValues may not sum up to $100 \%$ due to missing and rounded numbers.

$\ddagger$ AOR values were generated by the simultaneous entry of covariates in a logistic regression model; Hosmer-Lemeshow Goodness-of-Fit test $\chi^{2}=6 \cdot 9_{(\mathrm{df}=8)}$, $P=0.55$.

\&As defined by the Centers for Disease Control and Prevention BMI estimates and growth charts.

IThe number of calories the respondent should consume based on his/her age, height and weight as indicated in the US Department of Agriculture Nutrition Pyramid.

-Includes the responses: very important, important and somewhat important. 
When asked to rate the importance of having calorie postings at the point of purchase, $93 \%$ of the respondents thought that calorie information was 'important' and $86 \%$ thought that restaurants should be required to post this information on menu boards. When asked about their food selection intentions if calorie postings were available, $67 \%$ reported that they would choose food and drinks with fewer calories; 15\% would order the same amount of calories; and 13\% did not know how it would change their order. Interestingly, 5\% would order more calories. Among those who were overweight or obese (nearly half of all respondents), most (>86\%) thought calorie information should be posted on menu boards.

\section{Potential impact of menu labelling on food selection intentions}

Findings from our analyses (Table 2) indicate that respondents who would eat fewer calories if pointof-purchase calorie postings were available were more likely to be female, Latino, between the ages of 25 and 34 years or 55 and 75 years, obese and of the opinion that calorie information was important and should be posted. Education, knowledge of daily caloric requirements and the frequency of eating out were not statistically significant in multivariate modeling, with the HosmerLemeshow Goodness-of-Fit test statistics equaling 0.55.

\section{Discussion}

Our findings suggest that menu labelling may have a favourable effect on food selection intentions among public health clinic patients who are obese, female from select age groups and/or are Latino. Additionally, receptivity to calorie information on menu boards as measured by dichotomised ratings of importance appeared relatively high among these patients, suggesting that menu labelling strategies such as SB 1420 may have a sustainable impact in this population. Presently, only a paucity of studies has attempted to elucidate the correlates of food selection intentions among various groups of individuals who eat fast foods ${ }^{(4,5,12)}$. Prior studies, for example, have suggested that changes in food selection intentions (purchasing intentions) depend on such factors as individual's degree of concern about his/her weight (e.g. a young normal-weight man might be expected to be less affected by menu labelling than an overweight/ obese woman who is dieting), perceived caloric content of food items and perceived risk of chronic disease $\mathrm{e}^{(12-14)}$. In a study of 241 adults, provision of calorie information for food items for which participants were likely to underestimate calories resulted in decreased purchase likelihood of these items from $37 \%$ to $24 \%$ among those in the experimental group $v$. controls ${ }^{(5)}$. Similarly, a study in New York City found that among patrons who saw calorie information at the point of purchase in Subway restaurants, those who reported that the information affected their purchase choices, ordered, on average, ninety-nine fewer calories than those who said it did not ${ }^{(4)}$.

\section{Limitations}

The generalisability of the CNIS findings is subject to at least three limitations. First, the study sample is not representative of the general population in Los Angeles County; instead, the sample is more representative of uninsured or underinsured individuals who rely on public health clinics for selected services. However, to the best of our knowledge, this is the first study to evaluate clinic patients' receptivity to and intention to change food selections as a result of menu labelling. Second, self-report and -selection bias may have overestimated the respondents' intentions to change food selections if calorie postings were made available at the point of purchase; however, the effects of self-selection bias may have been mitigated by the survey's high response rate. Finally, the study design may be inadequate for evaluating other factors that may influence the degree of consumer response to menu labelling. Most experts would agree that menu labelling is a context-dependent intervention for which impact depends on other critical factors such as food industry marketing and simultaneous availability of tasty, competitively priced items (e.g. many lower priced foods are high in calories, fat, sugar and sodium) ${ }^{(12)}$.

\section{Conclusions}

Study findings suggest that patients of public health clinics are generally receptive to menu labelling and that they would order reduced calorie meals, if given calorie information at the point of purchase. These findings also suggest that the clinical setting may represent missed opportunities for raising awareness and educating consumers about how calorie postings in fast food and table service restaurants can improve their food selection and health, especially among low-income, uninsured or under-insured adults who often have poor diets and are at increased risk of preventable, diet-related, chronic health conditions. Corroborating evidence from the ongoing evaluation of SB 1420 after its implementation should provide further insights into whether some of these self-reported food selection intentions translate into action in this clinic-based population.

\section{Acknowledgements}

Sources of funding: The study was conducted as part of health assessment activities at the Los Angeles County Department of Public Health without external funding or support. Financial disclosure: None of the authors has any conflict of interest related to this work. Author contributions: All authors contributed to the analysis, 
interpretation and presentation of the data, and helped draft the article or revised it critically for important intellectual content. J.P., L.V.S. and P.S. conceptualised the initial study design that subsequently underwent iterative revisions. J.P. and L.V.S. were responsible for supervising data collection. P.L.C. was responsible for developing the logic framework used in the study. L.V.S. and T.K. provided statistical expertise and were responsible for developing the multivariate model. Ethical approval: The study received exemption approval from the Institutional Review Board (IRB) at the Los Angeles County Department of Public Health.

\section{References}

1. California Senate Bill 1420 (2008) Introduced by Senators Padilla and Migden on February 21, 2008; amended in the Assembly on August 8 and 22, 2008; amended in the Senate on May 13, 2008; passed September 2008. Document available at http://www.leginfo.ca.gov

2. Ogden CL, Carroll MD, Curtin LR, McDowell MA, Tabak CJ \& Flegal KM (2006) Prevalence of overweight and obesity in the United States, 1999-2004. JAMA 295, $1549-1555$.

3. Kuo T, Jarosz CJ, Simon P \& Fielding JE (2009) Menu labeling as a potential strategy for combating the obesity epidemic. A health impact assessment. Am J Public Health (Epublication ahead of print version).

4. Bassett MT, Dumanovsky T, Huang C, Silver LD, Young C, Nonas C, Matte TD, Chideya S \& Frieden TR (2008) Purchasing behavior and calorie information at fast-food chains in New York City, 2007. Am J Public Health 98, 1457-1459.

5. Burton S, Creyer EH, Kees J \& Huggins K (2006) Attacking the obesity epidemic: the potential health benefits of providing nutrition information in restaurants. Am J Public Health 96, 1669-1675.

6. US Census (2008) Los Angeles County, California. http:// factfinder.census.gov/home/saff/main.html?_lang=en (accessed November 2008)

7. Office of Health Assessment and Epidemiology (2007) Preventing Childhood Obesity: The Need to Create Healthy Places. A Cities and Communities Report. Los Angeles, CA: Los Angeles County Department of Public Health.

8. Simon PA, Kwan D, Angelescu A, Shih M \& Fielding JE (2008) Proximity of fast food restaurants to schools: do neighborhood income and type of school matter? Prev Med 47, 284-288.

9. Aday LA \& Cornelieus LJ (2006) Designing and Conducting Health Surveys: A Comprehensive Guide, 3rd ed. San Francisco, CA: Jossey-Bass.

10. United States Department of Agriculture (USDA) (2005) MyPyramid food intake pattern calorie levels for professionals. http://www.mypyramid.gov/professionals/pdf_ calorie_levels.html (accessed November 2008).

11. Becker M (1974) The Health Belief Model and personal health behavior. Health Educ Monogr 2 (entire issue).

12. Berman M \& Lavizzo-Mourey R (2008) Obesity prevention in the information age. Caloric information at the point of purchase. JAMA 300, 433-435.

13. Sproul AD, Canter DD \& Schmidt JB (2003) Does pointof-purchase nutrition labeling influence meal selections? A test in an Army cafeteria. Mil Med 168, 556-560.

14. Ludwig DS \& Nestle M (2008) Can the food industry play a constructive role in the obesity epidemic? JAMA 300, 1808-1813. 